\title{
Accuracy of digital templating of uncemented total hip arthroplasty at a certified arthroplasty center: a retrospective comparative study
}

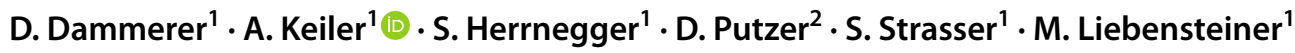

Received: 18 August 2020 / Accepted: 15 February 2021 / Published online: 16 March 2021

(c) The Author(s) 2021

\begin{abstract}
Introduction To investigate the accuracy of preoperative digital templating for total hip arthroplasty (THA) at a certified arthroplasty center (EndoCert EPZmax).

Materials and methods In a retrospective study design, we analysed 620 uncemented primary THAs for templating accuracy by comparing the preoperatively planned THA component size and the implanted size as documented by the surgeon. Templating was determined to be a) exact if the planned and the implanted component were the same size and b) accurate if they were exact \pm one size. Moreover, we investigated factors that potentially influence templating accuracy: overweight and obesity (WHO criteria), sex, implant design, surgeon experience, preoperative diagnosis. Digital templating was done with MediCAD software. The Mann-Whitney $U$ test and the Kruskal-Wallis test were used for statistical analysis.

Results Templating was exact in $52 \%$ of stems and $51 \%$ of cups and was accurate in $90 \%$ of the stems and $85 \%$ of the cups. Regarding the factors potentially influencing templating accuracy, the type of cup implant had a significant influence $(p=0.016)$. Moreover, greater accuracy of stem templating was achieved in female patients $(p=0.004)$. No such effect was determined for the other factors investigated.

Conclusions We conclude that preoperative 2D templating is accurate in $90 \%$ of the stems and $85 \%$ of the cups. Greater accuracy may be achieved in female patients. In addition to gender, the type of implant used may influence planning accuracy as well. Surgeon experience, BMI and preoperative diagnosis did not influence templating accuracy.

Level of evidence Level III (retrospective comparative study with prospective cohort).
\end{abstract}

Keywords Total hip arthroplasty $\cdot$ Digital templating $\cdot$ Experience $\cdot$ Accuracy $\cdot$ EndoCert

\section{Introduction}

Preoperative planning is an essential and integral part of the total hip arthroplasty (THA) procedure [1]. Digital templating of THA is well established, facilitates the determination of the correct implant size and helps restore the patientspecific physiological biomechanical conditions such as leg length, offset, center of rotation as well as lateralization [1-3]. Moreover, preoperative surgical planning improves

A. Keiler

alexander.keiler@i-med.ac.at

1 Department of Orthopaedics and Traumatology, Medical University of Innsbruck, Anichstrasse 35, 6020 Innsbruck, Austria

2 Department of Orthopaedics and Traumatology, Experimental Orthopaedics, Medical University of Innsbruck, Sonnenburgstrasse 16, 6020 Innsbruck, Austria postoperative range of motion and stability, shortens the operative time and reduces wear caused by malpositioning of the implant components [1-5].

A variety of factors that might negatively influence the accuracy of digital templating are mentioned in the current literature [4, 6-8]. Difficulties in determining the correct magnification factor for calibrating digital $\mathrm{X}$-rays have been described, especially for obese patients [4, 6-8]. In addition, better results affecting the accuracy of the predicted component size using digital preoperative planning software have been shown in combination with the planner's experience [1-4, 9-11]. According to the literature, even implant design seems to have an effect on planning accuracy [11].

There is evidence to support the issues and the extent to which digital planning matches the actual intraoperatively selected THA size $[1,7,12,13]$, but to the best of our knowledge our study is the first to investigate the accuracy of preoperative THA planning at an EndoCert EPZmax 
center $[14,15]$. At such a certified arthroplasty department, endoprosthetic interventions and preoperative templating are performed according to the guidelines and specifications of EndoCert [16, 17]. EndoCert is an initiative of the German Society for Orthopaedics and Orthopaedic Surgery (DGOOC) and is the world's first system for the certification of medical facilities in the field of joint replacement [14]. The EndoCert certification system is intended to ensure high-quality patient care and high patient safety in endoprosthetic procedures [16]. The process quality, interdisciplinary and constantly evolving treatment paths as well as a high level of training for all professional groups involved in the treatment are of central importance in the certification process $[14,17]$. In addition, certified surgeons have to perform a predetermined number of joint replacements per year [17]. Institutions that meet the requirements can be certified as an endoprosthetic center or endoprosthetic center for maximum care $[15,17]$.

We, therefore, aimed to (a) determine in how many cases preoperative templating accurately matched the implant size chosen intraoperatively and (b) analyse factors that might have influenced the accuracy of preoperative digital templating in patients who underwent THA in an EndoCert max center (experience, body mass index (BMI), sex, implant design and preoperative diagnosis).

\section{Materials and methods}

The study protocol was approved by the local ethics committee of the Medical University (No. 1150/2019) and performed in line with the principles of the Declaration of Helsinki.

We retrospectively investigated and included all patients who underwent primary THA at our department between January 2017 and August 2019. A total of 786 patients, giving a total of 843 implanted THAs, were investigated. Inclusion criteria were defined solely as the implantation of a primary uncemented total hip implant in the above-mentioned and defined period of time. Exclusion criteria were prior surgical interventions in the hip joint, previous fractures with joint involvement, cemented THA, revision surgery and intraoperative complications such as periprosthetic fractures and malalignment of the femoral stem in postoperative anterior-posterior X-rays (defined as $5^{\circ}<$ varus or valgus). A total of 578 patients met our inclusion criteria, resulting in 620 uncemented THAs.

Socio-demographic data and patient-related factors were collected to analyse a possible influence on the preoperative planning and the intraoperatively selected component size. These factors included body mass index (BMI), preoperative diagnosis, side of the operation, cut-to-suture time, positioning of the THA (cup: inclination and anteversion; stem: $5^{\circ}<$ varus or valgus), surgical approach as well as the planner's experience and intraoperative and postoperative complications. We included the uncemented implants most commonly used at our department:

Trident PSL cup in combination with the Accolade II stem (both Stryker Orthopaedics, Mahwah, NJ, USA) and the Pinnacle cup combined with the Corail stem (both DePuy Synthes, Warsaw, IN, USA). The Trident PSL cup is $1.8 \mathrm{~mm}$ wider than the stated size. This is meant to achieve an interference fit at the periphery of the implant $[18,19]$. The Accolade II has a morphologic wedge and a size-specific medial curvature [20]. The Trident-Accolade II THA was found to be a common implant combination in Germany. According to the annual report of the German Joint Replacement Registry, 1875 Trident PSL cups and 3086 Accolade II stems were implanted in Germany in 2018 [21].

The Pinnacle cup is a spherical cup with a single radius $[1,22]$. The Corail stem is designed to sit in the cancellous bone. It is hydroxyapatite-coated and has trapezoidal-like proximal cross-sections to provide rotational stability [1, 23]. According to the German Joint Replacement Registry in 2018 the Pinnacle cup was the most commonly used hip cup in Germany with a total of 17,878 implantations. The Corail stem was implanted 20046 times in Germany during the same period [21].

The decision for one or the other type of cup or stem was made by the surgeon himself, who preferred to use a particular cup or stem type, and did not depend on the patient. Thus, there is no selection bias for the implanted cup or stem component.

\section{Preoperative radiographs and digital templating}

All radiographs were taken with the same technique: anterior-posterior (AP) radiographs; patient standing in the upright position and full weight-bearing. The tube-to-film distance was standardised at $1.15 \mathrm{~m}$ by the Dept. of Radiology. A radiopaque ball with a diameter of $25 \mathrm{~mm}$ served as a size reference, which was placed in a standardised manner according to the manufacturer's recommendations: at the level of the femur shaft with symmetrical positioning of the pelvis, hip joints in a neutral position, longitudinal femur axis parallel to the image receptor plane, patella in zero position and central ray beam on femoral head center and symphysis respectively (Fig. 1) [24]. Preoperative digital templating was done with the MediCAD program (mediCAD Hectec GmbH, Altdorf/Landshut, Germany, Fig. 1). Thus, planning was performed by both specialized and nonspecialized hip surgeons. Surgeons certified by EndoCert were deemed experienced and specialized hip surgeons.

We investigated the cup and the stem separately. Accuracy of preoperative templating was determined by comparing the difference between planned and implanted component 
Fig. 1 Preoperative planning. Standardised antero-posterior view of the pelvis with a correctly placed calibration marker. MediCAD software

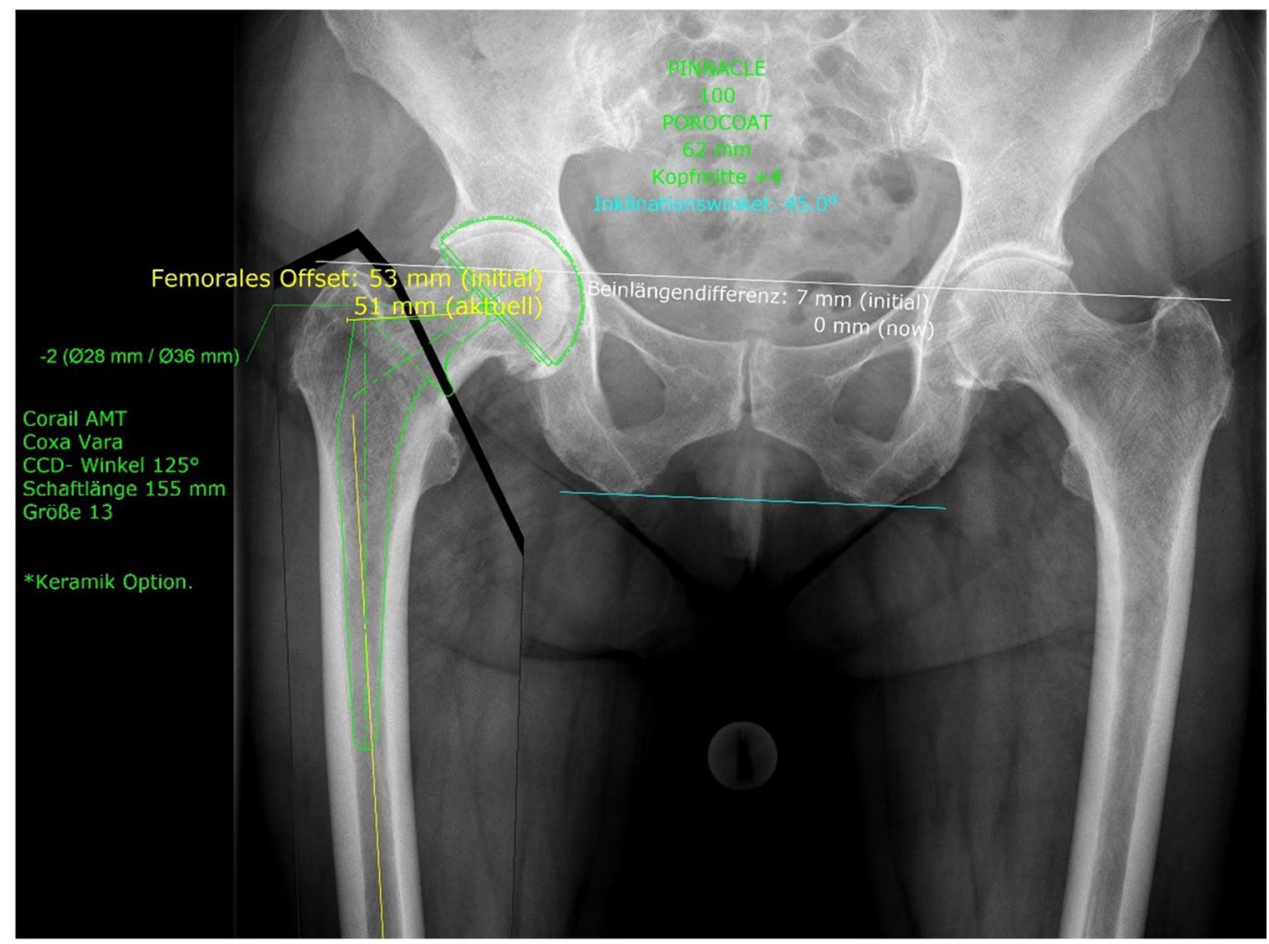

sizes as documented in the surgical report. If the planned and implanted THA components were the same size this was taken as 'exact'. A variance of $+/$ - one size was still considered to be accurate. Deviations of more than one size were considered inaccurate $[1,25,26]$.

\section{Statistical analysis}

Statistical analysis was performed with SPSS version 26 (IBM SPSS statistics, Chicago, IL, USA). Level of significance was set at $p<0.05$. Descriptive statistics were applied for sex, age, BMI, preoperative diagnosis and side of the operation. The influence that the planner's experience level, the component manufacturer and the patient's sex had on accuracy was analysed with the Mann-Whitney $U$ test. Correlation between BMI and planning accuracy was investigated with the Kruskal-Wallis test and paired post-hoc tests.

\section{Results}

A total of 620 (left: 277; right: 343) cementless THAs in 578 patients (female: 298; male: 280) were investigated. Mean age at surgery was 66.1 (range: 16.7-90.3) years. In 578 cases the THA was performed on one side and in 42 cases on both sides simultaneously. Mean body mass index was 26.7 (range: 16.9-59.2) $\mathrm{kg} / \mathrm{m}^{2}$. According to the definition of the World Health Organization (WHO), 207 participants were under- or normal weight (BMI up to 24.9), 255 were overweight (BMI between 25 and 30) and 157 were obese (BMI from 30.1 upwards). The most common indication for THA was primary osteoarthritis in 533 out of 620 cases. In 56 cases necrosis of the femoral head, in 29 hip dysplasia and in 12 cases protrusion osteoarthritis of the hip was the indication for THA surgery. Mean cut-to-suture time was 67.7 (range 37-181) minutes. In all patients, a direct anterior approach was performed for the THA procedure [27, 28]. A Trident PSL cup and an Accolade II stem were implanted in $88 \%(n=544)$, and a Pinnacle cup and Corail stem were used in $12 \%(n=76)$ of the procedures. Details are given in Table 1 and Fig. 2.

\section{General templating accuracy}

For the cups, templating was found to be exact in $51 \%$ of our cases $(n=315)$. In 34\% ( $n=208)$, preoperative templating differed by one size. For the stems, templating was found to be exact at $52 \%(n=320)$. In $38 \%(n=256)$ the preoperative templating of the stems differed by one size. In other words, in total $85 \%(n=523)$ of the cups and 90\% $(n=576)$ of the stems were calculated accurately (exact \pm one size). Details are given in Table 2 .

\section{Factors with potential influence on templating accuracy}

A total of 544 Trident PSL cups were investigated. Of the Trident PSL cups 49\% ( $n=267)$ were planned exactly, and 
Table 1 Demographic data of patients, distribution of BMD and primary diagnosis, mean duration of the operation and used implants

\begin{tabular}{ll}
\hline Number of patients & \\
Female & 298 \\
Male & 280 \\
Total & 578 \\
Operated side & \\
Left & 277 \\
Right & 343 \\
Total & 620 \\
Mean age in years & $66.1(16.7-90.3)$ \\
Mean BMI & $26.7(16.9-59.2)$ \\
BMI (WHO classification in $\left.\mathrm{kg} / \mathrm{m}^{2}\right)$ & \\
Under- or normal weight & 207 \\
Overweight & 255 \\
Obese & 157 \\
Preoperative diagnosis & \\
Primary osteoarthritis & 533 \\
Protrusion osteoarthritis & 12 \\
Femoral head necrosis & 56 \\
Hip dysplasia & 19 \\
Average duration of surgery in minutes & $67.7(37-181)$ \\
Number of implanted cups & \\
Trident PSL & 544 \\
Pinnacle & 76 \\
Number of implanted stems & \\
Accolade II & 544 \\
Corail & 76 \\
\hline
\end{tabular}

in $35 \%(n=187)$ accuracy was within \pm one size. Of the Pinnacle cups $63 \%(n=48)$ were planned exactly and $28 \%$ $(n=21)$ accurately. Thus, the Trident PSL cup was templated accurately in $84 \%(n=454)$ of our cases, and the Pinnacle cup was planned accurately in $91 \%(n=69)$. The difference between the two cup types was statistically significant $(p=0.016)$. A total of 540 Accolade II stems were analysed. In 53\% $(n=288)$ preoperative planning corresponded exactly to the implanted stem size. In $38 \%(n=202)$ of the stems planning differed by \pm one size. A total of 76 Corail stems were analysed: $42 \%$ of the stems $(n=32)$ were planned exactly and in $43 \%(n=33)$ the preoperative planning was accurate. Thus, the Accolade II stem was templated accurately in $91 \%(n=490)$ of our procedures, and the Corail stem was accurately planned in $86 \%(n=65)$. No statistically significant difference was found in the accuracy of the preoperative planning of the Accolade II as compared to that of the Corail stem ( $p=0.052$, Table 2$)$.

Regarding surgeon experience as a factor potentially influencing templating accuracy, it was found that certified arthroplasty surgeons achieved accuracy in $90 \%(n=150)$ of cases. Non-certified surgeons accurately planned the cup in $85 \%(n=79)$ and the femoral stem in $86 \%(n=80)$ of procedures. No significant difference was found (cup: $p=0.353$; stem: $p=0.169$ ) (Table 2).

The planning precision in the different BMI groups, according to the definition of the WHO, showed in the group of the normal to underweight patients an accuracy of $83 \%$ $(n=171)$ for the cup and 91\% $(n=191)$ for the stem. In the group of overweight participants, the cup was templated accurately in $87 \%(n=221)$ and the stem in $91 \%(n=237)$. In the group of obese patients in $83 \%(n=130)$ the cup and in $79 \%(n=141)$ the stem was planned accurately. No statistical significance was found ( $p=0.422$ for the cup; $p=0.216$ for the stem) (Table 2).

We found a statistically significant difference in templating accuracy for the femoral stem between the two sexes $(p=0.004)$. Planning accuracy was seen to be greater in women than in men. The stem was planned accurately in 94\% $(n=295)$ of implants in the female patients and in $86 \%$ $(n=260)$ in the male patients. Templating accuracy of the cup did not significantly differ between the sexes $(p=0.602)$. In $86 \%(n=259)$ of the men and $83 \%(n=264)$ of the women the cup component was templated accurately.

Regarding a potential effect of the type of diagnosis on templating accuracy, no statistically significant results were found ( $p=0.176$ for the cup, $p=0.354$ for the stem). In patients with primary osteoarthritis accuracy was reached in $84 \%$ for the cup ( $n=448)$ and in $89 \%$ for the stem $(n=475)$. In protrusion osteoarthritis, $92 \%(n=11)$ of the cups and $100 \%(n=12)$ of the stems were predicted accurately. In femoral head necrosis $93 \%(n=50)$ of the cups and the stems were planned accurately. In patients with hip dysplasia templating was accurate in $74 \%(n=14)$ of cups and in $90 \%$ $(n=18)$ of stems (Table 2$)$.

\section{Discussion}

The most important findings of the study were that templating was found to be accurate in $90 \%$ of the stems and in $85 \%$ of the cups. Regarding the investigated factors that potentially influenced templating accuracy, it was found that the type of cup implant had a significant influence $(p=0.016)$. Moreover, greater accuracy of stem templating was achieved in female patients $(p=0.004)$. For the rest of the investigated factors no such effect was determined.

When comparing our findings with those of previous research it appears that Holzer et al. analysed 632 preoperatively planned uncemented THAs, of which the cup and the stem were preoperatively determined to be within one size in $78 \%$ and in $87 \%$, respectively [1]. In the study by Whiddon et al., planning accuracy was shown to be $78 \%$ for the acetabular and $90 \%$ for the femoral component (both within \pm one implant size) [26]. Sershon et al. showed an accuracy of digital templating within two sizes of the final 
Fig. 2 Flowchart of the study group constellation

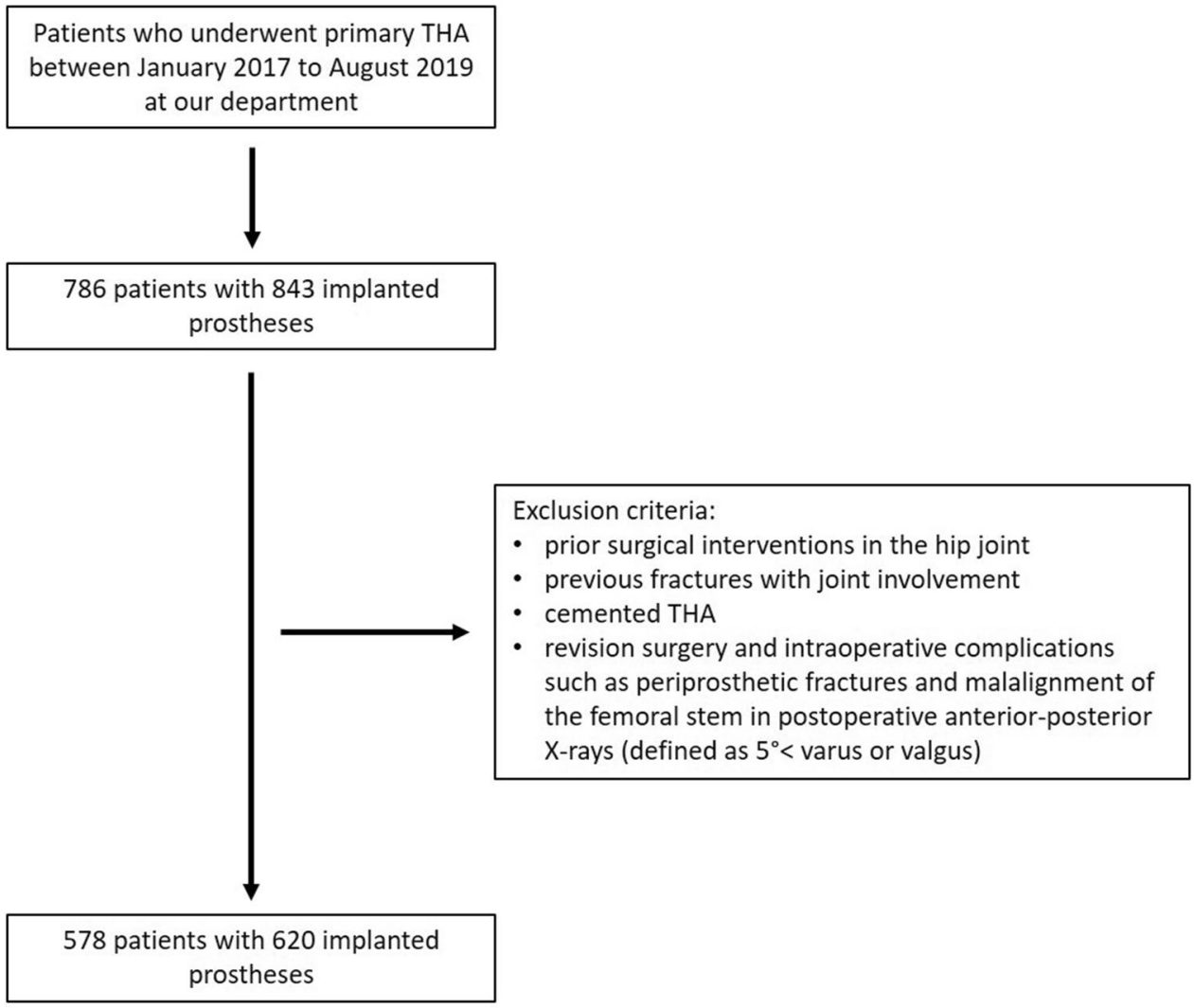

acetabular and femoral implants in $99.1 \%$ and in $97.1 \%$ of cases, respectively [25]. However, it may be questioned whether \pm two implant sizes should still be taken as accurate. Wiese et al. showed a planning accuracy of $71 \%$ for the acetabular and $79 \%$ for the femoral component [29]. Roughly, the results of the current investigation match those of the studies mentioned above (Table 3 ).

Underlying studies, like the present work, are preoperative predictions based on two-dimensional electronic $\mathrm{X}$-ray images. However, today there are numerous other options for preoperative planning in THA. Studies using CT-based three-dimensional planning show a significantly higher planning accuracy than do preoperative predictions based on two-dimensional electronic X-ray images. Sariali et al. and Osmani et al. demonstrated an accuracy of more than $95 \%$ when planning was based on three-dimensional CT images [30, 31]. The three-dimensional imaging based on CT images provides surgeons with more bone structures to assist in planning and increase accuracy [32]. Schiffner et al. were able to demonstrate the superiority of CT-based three-dimensional planning over 2D planning but emphasized that greater planning accuracy did not necessarily mean better clinical outcome [33]. Additionally, nowadays it is possible to plan automatically using CT imagination. Kagiyama et al. developed a system that is able to determine the most suitable implant by collecting data from an experienced surgeon [34]. Nevertheless, CTbased planning is still controversial. While Rübberdt et al. pointed out the greater radiation exposure in the area of the gonads, Henckel et al. argued that radiation exposure is negligible if CT scans are performed with special low-dose recordings [35, 36]. Furthermore, technical opportunities for preoperative three-dimensional imaging based on CT scans are not given in every hospital and sufficient precision can also be achieved with two-dimensional planning methods. Although the methods of CT-based three-dimensional and computer-assisted planning are manifold, the individual orthopaedist achieves the safest results with the method in which he was trained and has experience [37]. Thus, two-dimensional X-ray-based planning is still the most widely used in clinical practice. Though there is a manifold number of two-dimensional planning software, technically the majority is based on the same process. Preoperative radiographs are taken to a standard anteroposterior view of the pelvis and a calibration object of individual size is placed between the legs of the patients. The digital templating software automatically calibrates the image and template-overlays according to the known size of the marker. Most clinics have switched from planning with analogue solid templates to digital planning, and therefore the current literature clearly shows that digital two-dimensional planning is well established [4]. 
Table 2 Planning accuracy and deviation of implants in absolute values and percentage

\begin{tabular}{|c|c|c|c|c|}
\hline & \multicolumn{2}{|l|}{ Cup size } & \multicolumn{2}{|l|}{ Stem size } \\
\hline & Occurrence & Percentage & Occurrence & Percentage \\
\hline \multicolumn{5}{|l|}{ Implant size in general } \\
\hline Exact match & 315 & 51 & 320 & 52 \\
\hline \pm 1 size & 208 & 34 & 256 & 38 \\
\hline \pm 2 sizes & 66 & 11 & 35 & 7 \\
\hline \pm 3 sizes or more & 31 & 5 & 9 & 3 \\
\hline \multicolumn{5}{|l|}{ Implant size Stryker } \\
\hline Exact match & 267 & 49 & 288 & 53 \\
\hline \pm 1 size & 187 & 35 & 202 & 38 \\
\hline \pm 2 sizes & 60 & 11 & 35 & 7 \\
\hline \pm 3 sizes or more & 30 & 5 & 15 & 3 \\
\hline \multicolumn{5}{|l|}{ Implant size DePuy Synthes } \\
\hline Exact match & 48 & 63 & 32 & 42 \\
\hline \pm 1 size & 21 & 28 & 33 & 43 \\
\hline \pm 2 sizes & 6 & 8 & 10 & 13 \\
\hline \multirow[t]{2}{*}{ \pm 3 sizes or more } & 1 & 1 & 1 & 1 \\
\hline & \multicolumn{2}{|c|}{ Accurate acetabular planning } & \multicolumn{2}{|c|}{ Accurate femoral planning } \\
\hline \multicolumn{5}{|l|}{ Planner's experience } \\
\hline EndoCert-certified surgeon & 150 & 90 & 150 & 90 \\
\hline Non-EndoCert-certified surgeon & 79 & 85 & 80 & 86 \\
\hline \multicolumn{5}{|l|}{ Sex } \\
\hline Female & 264 & 83 & 295 & 94 \\
\hline Male & 259 & 86 & 260 & 86 \\
\hline \multicolumn{5}{|l|}{ Preoperative diagnosis } \\
\hline Primary osteoarthritis & 448 & 84 & 475 & 89 \\
\hline Protrusion osteoarthritis & 11 & 92 & 12 & 100 \\
\hline Femoral head necrosis & 50 & 93 & 50 & 93 \\
\hline Hip dysplasia & 14 & 74 & 18 & 90 \\
\hline
\end{tabular}

Deviation of one size is considered to be accurate

The presented study analysed the Trident PSL and the Pinnacle acetabular component systems and demonstrated that the Pinnacle cup achieved greater templating accuracy than did the Trident PSL cup $(p=0.016)$. When comparing the different femoral components, no significant difference was seen between the Accolade II and the Corail stem $(p=0.052)$. One possible explanation might be found in the "peripheral self-locking" system of the Trident PSL cup. The outside diameter of the cup is $1.8 \mathrm{~mm}$ wider than the actual reamed size. The surgeon has to take this into consideration when planning the cup size as well as intraoperatively when reaming the cup [18]. Furthermore, the differences in bone stock from patient to patient might have an influence, as surgical protocols mention that reaming should be performed with special attention to bone quality [18].

The presented study showed a tendency to predicting the acetabular component too large (30\% planned too large, $19 \%$ too small), which coincides with the results reported by Wiese et al., where a tendency to estimate both components too large was proven [29].

Several studies have reported the experience of the orthopaedist who performed the planning to be a significant factor influencing the accuracy of preoperative planning. Carter et al. demonstrated a significant impact on the planning of both components, Holzer et al. only for the femoral component $[1,38]$. Efe et al. and Strøm et al. showed no significant influence of the experience of the planning orthopaedic surgeon [39, 40], which is in line with the findings of the present study $(p=0.353$ for the acetabular component; $p=0.169$ for the femoral component). This might be due to the fact that the study population could possibly be too small since the planning surgeon could be identified in only $42 \%$ of the performed operations. However, the results show a trend, according to which specialized arthroplasty surgeons are more precise in their preoperative planning. With a larger 
Table 3 Overview of comparable literature

\begin{tabular}{|c|c|c|c|c|c|c|c|}
\hline Study & Year & $\begin{array}{l}\text { Num- } \\
\text { ber of } \\
\text { patients }\end{array}$ & Implants cup & Implants stem & $\begin{array}{l}\text { General accuracy } \\
\text { cup (exact size } \pm 1 \\
\text { size) }\end{array}$ & $\begin{array}{l}\text { General } \\
\text { accuracy stem } \\
\text { (exact size } \pm 1 \\
\text { size) }\end{array}$ & Software \\
\hline Holzer et. al. [1] & 2019 & 632 & $\begin{array}{l}\text { Allofit } \AA \\
\text { Pinnacle } \AA\end{array}$ & $\begin{array}{l}\text { Alloclassic } ₫ \\
\text { Corail } ₫\end{array}$ & $78 \%(n=494)$ & $87 \%(n=547)$ & $\begin{array}{l}\text { EndoMap software }{ }^{\circledR} \\
\text { system (Siemens Medical } \\
\text { Solutions AG, Erlangen, } \\
\text { Germany) }\end{array}$ \\
\hline Eggli et. al. [2] & 1998 & 100 & n.a & $\begin{array}{l}\text { Müller® } \\
\text { straight }\end{array}$ & $90 \%(n=90)$ & $92 \%(n=92)$ & $\begin{array}{l}\text { Software developed by } \\
\text { Maurice E. Müller Foun- } \\
\text { dation (Bern, Switzer- } \\
\text { land) and by Department } \\
\text { of Bioengineering Clem- } \\
\text { son University (South } \\
\text { Carolina, USA) }\end{array}$ \\
\hline Davila et. al. [7] & 2006 & 36 & Pinnacle ${ }^{\circledR}$ & Summit® & $86 \%(n=31)$ & $72 \%(n=26)$ & $\begin{array}{l}\text { EndoMap® software } \\
\text { system (Siemens Medical } \\
\text { Solutions AG, Erlangen, } \\
\text { Germany) }\end{array}$ \\
\hline Gamble et. al. [12] & 2010 & 40 & Trident $®$ & $\begin{array}{l}\text { Accolade }{ }^{\circledR} \\
\text { Omnifit }{ }^{\circ}\end{array}$ & $80 \%(n=32)$ & $85 \%(n=34)$ & $\begin{array}{l}\text { OrthoView }{ }^{\circledR} \text { software } \\
\text { (version 2.0CEN, } \\
\text { Meridian Technique Ltd, } \\
\text { Southampton, United } \\
\text { Kingdom) }\end{array}$ \\
\hline $\begin{array}{l}\text { Shaarani et. al. } \\
\text { [13] }\end{array}$ & 2013 & 100 & Trident $®$ & Accolade ${ }^{\circledR}$ & $80 \%(n=80)$ & $98 \%(n=98)$ & $\begin{array}{l}\text { OrthoView@ software } \\
\text { (version 2.0CEN, } \\
\text { Meridian Technique Ltd, } \\
\text { Southampton, United } \\
\text { Kingdom) }\end{array}$ \\
\hline $\begin{array}{l}\text { Whiddon et. al. } \\
\text { [26] }\end{array}$ & 2011 & 51 & Trident $®$ & $\begin{array}{l}\text { Secur-Fit Max } \AA \\
\text { Accolade } \AA\end{array}$ & $78 \%(n=40)$ & $90 \%(n=46)$ & $\begin{array}{l}\text { Impax }{ }^{\circledR} \text { digital templating } \\
\text { software (Agfa, Mortsel, } \\
\text { Belgium) }\end{array}$ \\
\hline Wiese et. al. [29] & 2020 & 56 & Pinnacle ${ }^{\circledR}$ & Summit ${ }^{\circ}$ & $71 \%(n=40)$ & $79 \%(n=44)$ & $\begin{array}{l}\text { Impax Orthopaedic Tools }{ }^{\circledR} \\
\text { software (Agfa, Mortsel, } \\
\text { Belgium) }\end{array}$ \\
\hline Carter et. al. $[38]^{\mathrm{a}}$ & 1995 & 74 & - & Osteonics ${ }^{\circledR}$ & - & $\begin{array}{l}82-96 \% \\
\quad(n=61-71)\end{array}$ & n.a \\
\hline Efe et. al. [39] & 2011 & 169 & $\begin{array}{l}\text { EP-FIT-PLUS® } \\
\text { Wagner® }\end{array}$ & $\begin{array}{l}\text { Polar }{ }^{\circledR} \\
\text { Proxy Plus }{ }^{\circledR}\end{array}$ & $78 \%(n=132)$ & $82 \%(n=139)$ & $\begin{array}{l}\text { MediCAD® software } \\
\text { (version } 2.06 \text {, mediCAD } \\
\text { Hectec GmbH, Altdorf/ } \\
\text { Landshut, Germany) }\end{array}$ \\
\hline
\end{tabular}

${ }^{a}$ Only accuracy of implanted stems was investigated. Accuracy between less ore more experienced surgeons was compared, no overall accuracy

study population, this might be a statistically significant influence.

No statistically significant impact of BMI was found on the accuracy of preoperative planning ( $p=0.422$ for the acetabular component; $p=0.216$ for the femoral component). Similarly, Sershon et al. showed no significant effect of BMI on templating accuracy [25]. Whiddon et al. divided the patients into obese and non-obese patients $(\mathrm{BMI} \geq / \leq 30)$ and did not assess any differences in the accuracy of planning $[25,26]$. In contrast, Holzer et al. showed a difference between normal- and overweight patients (BMI 18.5-24.9 for normal-weight patients or 25-29.9 for overweight patients) regarding accuracy [1].
In the presented study, significantly greater precision in preoperative planning was observed in female patients than in male patients, but only for the femoral component $(p=0.004)$ and not for the acetabular component $(p=0.602)$. Holzer et al. found no significant difference in the accuracy of preoperative planning between sexes [1].

Templating with accurate and reliable calibration markers is of the utmost importance in THA, as it has been shown that calibration errors using external calibration markers significantly influence component sizes [41]. In a recent study, Warschawski et al. compared the accuracy in the preoperative component selection of the double marker (King Mark) method, which may be more accurate than 
a single marker method, with the conventional metal ball method in the general population and in obese patients. However, the study found no difference between the King Mark method and the conventional metal ball method in the ability to accurately predict component sizes. In the subgroup of obese patients, the King Mark technique offered no advantage for accurately predicting component sizes [42].

Recently, Kase et al. presented a classification system to aid surgeons during their preoperative analysis, outlining the importance of considering femoral head translation during preoperative templating [43]. The authors describe a classification system to distinguish five types of architectural hip deformities, based on femoral head translation patterns, and advise surgeons to adapt their templating strategy accordingly. In a consecutive study, the authors evaluated whether mismatch between planned and real implant sizes compromises THA outcomes [44]. According to the given results, implanting a component of a different size than planned seemed not to compromise THA outcomes in terms of the Forgotten Joint Score (FJS) and Oxford Hip Score (OHS). Therefore, the authors advise that surgeons should respect their intraoperative findings when it comes to the ultimate implant size selection.

The following limitations are acknowledged. There was a lack of variability in the study population and therefore the subgroups were too small. Thus, the accuracy in planning for the different underlying diagnoses is not sufficiently comparable (osteoarthritis, dysplasia, femoral head necrosis etc.). Another limiting factor is the small amount of information available about the planning surgeon due to the retrospective character of the study. Of 620 included hip prostheses only 260 planning surgeons could be assessed. The study demonstrates a trend, according to which planning accuracy tends to increase with the experience of the planning surgeon, but no statistical significance was found ( $p=0.353$ for the cup; $p=0.169$ for the stem). With a larger study population, there might be a relevant possibility to detect a significant difference.

\section{Conclusions}

From our findings, we conclude that preoperative 2D templating is accurate in $90 \%$ of the stems and $85 \%$ of the cups. In female patients, greater accuracy may be achieved. In addition to gender, the type of implant used may influence planning accuracy as well. Surgeon experience, BMI and preoperative diagnosis did not influence templating accuracy.
Funding Open access funding provided by University of Innsbruck and Medical University of Innsbruck. This research did not receive any specific grant from funding agencies in the public, commercial, or not-for-profit sectors.

\section{Compliance with ethical standards}

Conflict of interest The authors declare that they have no conflicts of interests.

Ethical approval This study was performed in line with the principles of the Declaration of Helsinki, approved by the Ethics Committee of the Medical University of Innsbruck (No. 1150/2019) and registered in a study register (No. 20191007-2113).

Consent to participate Written informed consent was obtained from all subjects before participation.

Open Access This article is licensed under a Creative Commons Attribution 4.0 International License, which permits use, sharing, adaptation, distribution and reproduction in any medium or format, as long as you give appropriate credit to the original author(s) and the source, provide a link to the Creative Commons licence, and indicate if changes were made. The images or other third party material in this article are included in the article's Creative Commons licence, unless indicated otherwise in a credit line to the material. If material is not included in the article's Creative Commons licence and your intended use is not permitted by statutory regulation or exceeds the permitted use, you will need to obtain permission directly from the copyright holder. To view a copy of this licence, visit http://creativecommons.org/licenses/by/4.0/.

\section{References}

1. Holzer LA, Scholler G, Wagner S et al (2019) The accuracy of digital templating in uncemented total hip arthroplasty. Arch Orthop Trauma Surg 139:263-268. https://doi.org/10.1007/ s00402-018-3080-0

2. Eggli S, Pisan M, Müller ME (1998) The value of preoperative planning for total hip arthroplasty. J Bone Jt Surg Ser B 80:382390. https://doi.org/10.1302/0301-620X.80B3.7764

3. Hoikka V, Paavilainen T, Lindholm TS et al (1987) Measurement and restoration of equality in length of the lower limbs in total hip replacement. Skeletal Radiol 16:442-446. https://doi.org/10.1007/ BF00350537

4. Della Valle AG, Padgett DE, Salvati EA (2005) Preoperative planning for primary total hip arthroplasty. J Am Acad Orthop Surg 13:455-462

5. Claes L, Kirschner P, Perka C, Rudert M (2012) AE-Manual der Endoprothetik: Hüfte und Hüftrevision. Springer-Verlag, Berlin

6. Franken M, Grimm B, Heyligers I (2010) A comparison of four systems for calibration when templating for total hip replacement with digital radiography. J Bone Jt Surg Ser B 92:136-141. https:// doi.org/10.1302/0301-620X.92B1.22257

7. Davila JA, Kransdorf MJ, Duffy GP (2006) Surgical planning of total hip arthroplasty: Accuracy of computer-assisted EndoMap software in predicting component size. Skeletal Radiol 35:390393. https://doi.org/10.1007/s00256-006-0106-4

8. Sinclair VF, Wilson J, Jain NPM, Knowles D (2014) Assessment of accuracy of marker ball placement in pre-operative templating for total hip arthroplasty. J Arthroplasty 29:1658-1660. https:// doi.org/10.1016/j.arth.2014.03.013 
9. Marston RA, Cobb AG, Bentley G (1996) Stanmore compared with Charnley total hip replacement: a prospective study of 413 arthroplasties. J Bone Jt Surg Ser B 78:178-184. https://doi.org/ 10.1302/0301-620X.78B2.0780178

10. Inglis T, Dalzell K, Hooper G et al (2013) Does orthopedic training compromise the outcome in total hip arthroplasty? J Surg Educ 70:76-80. https://doi.org/10.1016/j.jsurg.2012.08.003

11. Jung S, Neuerburg C, Kappe T et al (2012) Validität der computergestützten Hü fttotalendoprothesenplanung: Einfluss von Schaftdesign und Untersuchererfahrung. Z Orthop Unfall 150:404-408. https://doi.org/10.1055/s-0031-1298386

12. Gamble P, de Beer J, Petruccelli D, Winemaker M (2010) The accuracy of digital templating in uncemented total hip arthroplasty. J Arthroplasty 25:529-532. https://doi.org/10.1016/j.arth. 2009.04.011

13. Shaarani SR, McHugh G, Collins DA (2013) Accuracy of digital preoperative templating in 100 consecutive uncemented total hip arthroplasties. A single surgeon series. J Arthroplasty 28:331337. https://doi.org/10.1016/j.arth.2012.06.009

14. Über EndoCert-EndoCert-Zertifizierte Endoprothetikzentren (2020). https://endocert.de/ueber-uns/ueber-endocert. Accessed 27 Jul 2020

15. Maßgebende Unterschiede EPZ und EPZmax. https://www.clarc ert.com/systeme/endoprothetikzentrum/system/ablauf/unterschie de-epz-und-epzmax.html. Accessed 14 Feb 2021

16. Ziele von EndoCert-EndoCert-Zertifizierte Endoprothetikzentren (2020). https://endocert.de/ueber-uns/ziele-von-endocert. Accessed 27 Jul 2020

17. Ablauf einer Zertifizierung - EndoCert - Zertifizierte Endoprothetikzentren. https://endocert.de/fuer-kliniken/ablauf-einer-zertifizie rung. Accessed 05 Feb 2021

18. Trident $®$ Acetabular System PSL Surgical Protocol. Indications for Trident Ceramic Insert with Ceramic Head. https://www.stryk ermeded.com/media/1157/trident-acetabular-system-psl-surgicalprotocol.pdf. Accessed 27 July 2020

19. Nunag P, Deakin AH, Oburu E, Sarungi M (2012) Two-year radiologic assessment of the trident peripheral self-locking cup using EBRA. HIP Int 22:511-515. https://doi.org/10.5301/HIP.2012. 9744

20. Design rationale Accolade ® II Femoral Hip Stem. http://az621 074.vo.msecnd.net/syk-mobile-content-cdn/global-content-system/SYKGCSDOC-2-44949/5UEhn7UHILqfWt6AV2yTIW2Ya mQiUg/ACCII_PG_3.pdf. Accessed 27 July 2020

21. Grimberg A, Jansson V, Melsheimer O, Steinbrück A (2019) Endoprothesenregister Deutschland [EPRD] - Jahresbericht 2019. https://www.eprd.de/fileadmin/user_upload/Jahresbericht_2019_ doppelseite_2.0.pdf. Accessed 04 Feb 2021

22. Surgical Techniques PINNACLE ® Hip Solutions DePuy Synthes. https://www.corailpinnacle.net/sites/default/files/2016-04/pinna cle-surgical-technique_0.pdf. Accessed 27 July 2020

23. DePuy Synthes CORAIL ${ }^{\circledR}$ Hip System Product Rationale and Surgical Technique. https://www.corailpinnacle.net/sites/default/ files/DSEMJRC061606652CORAIL\%20Platform\%20Brochure. pdf. Accessed 27 July 2020

24. mediCAD® V6.5 Image Requirements and Scaling. https://www. medicad.eu/en/service-support/medicad-books/summary/2-medic ad-handbuecher/3-medicad-manual-2020-en. Accessed 14 Feb 2021

25. Sershon RA, Diaz A, Bohl DD, Levine BR (2017) Effect of body mass index on digital templating for total hip arthroplasty. $\mathbf{J}$ Arthroplasty 32:1024-1026. https://doi.org/10.1016/j.arth.2016. 09.020

26. Whiddon DR, Bono JV, Lang JE et al (2011) Accuracy of digital templating in total hip arthroplasty. Am J Orthop (Belle Mead NJ) 40:395-398
27. Rachbauer F (2005) Minimally invasive total hip arthroplasty via direct anterior approach. Orthopade 34:1103-1104. https://doi. org/10.1007/s00132-005-0854-1

28. Meermans G, Konan S, Das R et al (2017) The direct anterior approach in total hip arthroplasty a systematic review of the literature. Bone Jt J 99B:732-740

29. Wiese K, Kock F, Blake C et al (2020) The accuracy of pre-operative digital templating in total hip arthroplasty performed in a low-volume, resource-constrained orthopaedic unit. SA Orthop J 19:28-32. https://doi.org/10.17159/2309-8309/2020/v19n1a4

30. Sariali E, Mauprivez R, Khiami F et al (2012) Accuracy of the preoperative planning for cementless total hip arthroplasty. A randomised comparison between three-dimensional computerised planning and conventional templating. Orthop Traumatol Surg Res 98:151-158. https://doi.org/10.1016/j.otsr.2011.09.023

31. Osmani FA, Thakkar S, Ramme A et al (2017) Variance in predicted cup size by 2-dimensional vs 3-dimensional computerized tomography-based templating in primary total hip arthroplasty. Arthroplast Today 3:289-293. https://doi.org/10.1016/j.artd.2016. 09.003

32. Colombi A, Schena D, Castelli CC (2019) Total hip arthroplasty planning. EFORT Open Rev 4:626-632. https://doi.org/10.1302/ 2058-5241.4.180075

33. Schiffner E, Latz D, Jungbluth P et al (2019) Is computerised 3D templating more accurate than $2 \mathrm{D}$ templating to predict size of components in primary total hip arthroplasty? HIP Int 29:270 275. https://doi.org/10.1177/1120700018776311

34. Kagiyama Y, Otomaru I, Takao M et al (2016) CT-based automated planning of acetabular cup for total hip arthroplasty (THA) based on hybrid use of two statistical atlases. Int J Comput Assist Radiol Surg 11:2253-2271. https://doi.org/10.1007/ s11548-016-1428-x

35. Rübberdt A, Wich M, Mutze S (2003) Digitale präoperative Planung von Hüftgelenkendoprothesen. Trauma und Berufskrankheit 5:315-321. https://doi.org/10.1007/s10039-003-0779-z

36. Henckel J, Richards R, Lozhkin K et al (2006) Very low-dose computed tomography for planning and outcome measurement in knee replacement. The imperial knee protocol. J Bone Jt Surg Ser B 88:1513-1518. https://doi.org/10.1302/0301-620X.88B11. 17986

37. Mainard D, Barbier O, Knafo Y et al (2017) Accuracy and reproducibility of preoperative three-dimensional planning for total hip arthroplasty using biplanar low-dose radiographs : a pilot study. Orthop Traumatol Surg Res 103:531-536. https://doi.org/10. 1016/j.otsr.2017.03.001

38. Carter LW, Stovall DO, Young TR (1995) Determination of accuracy of preoperative templating of noncemented femoral prostheses. J Arthroplasty 10:507-513. https://doi.org/10.1016/S08835403(05)80153-6

39. Efe T, El Zayat BF, Heyse TJ et al (2011) Precision of preoperative digital templating in total hip arthroplasty. Act Orthop Belg 77:616-621

40. Strøm NJ, Pripp AH, Reikerås O (2017) Templating in uncemented total hip arthroplasty-on intra- and interobserver reliability and professional experience. Ann Transl Med. https://doi.org/10. 21037/atm.2017.01.73

41. Boese CK, Wilhelm S, Haneder S et al (2019) Influence of calibration on digital templating of hip arthroplasty. Int Orthop 43:1799 1805. https://doi.org/10.1007/s00264-018-4120-7

42. Warschawski Y, Shichman I, Morgan S et al (2020) The accuracy of external calibration markers in digital templating using the double marker and single marker method: a comparative study. Arch Orthop Trauma Surg 140:1559-1565. https://doi.org/10.1007/ s00402-020-03569-2

43. Kase M, O'Loughlin PF, Ait-Si-Selmi T et al (2020) Pre-operative templating in THA. Part I: a classification of architectural hip 
deformities. Arch Orthop Trauma Surg 140:129-137. https://doi. org/10.1007/s00402-019-03298-1

44. Cech A, Kase M, Kobayashi H et al (2020) Pre-operative planning in THA. Part III: do implant size prediction and offset restoration influence functional outcomes after THA? Arch Orthop Trauma Surg 140:563-573. https://doi.org/10.1007/s00402-020-03342-5
Publisher's Note Springer Nature remains neutral with regard to jurisdictional claims in published maps and institutional affiliations. 